\title{
The mutual relationship between heart failure and atrial fibrillation
}

\author{
Filippo M. Sarullo ${ }^{1}$, Giuseppe Schembri ${ }^{1}$, Cinzia Nugara ${ }^{1,2}$, Silvia Sarullo ${ }^{3}$, Giuseppe Vitale ${ }^{1}$, \\ Salvatore Corrao ${ }^{4}$
}

${ }^{1}$ Cardiovascular Rehabilitation Unit, Buccheri La Ferla Fatebenefratelli Hospital, Palermo; ${ }^{2}$ Biomedical Department of Internal Medicine and Specialities (DIBIMIS), University of Palermo, IRCSS Bonino Pulejo, Messina; ${ }^{3}$ Cardiology Unit, University of Palermo; ${ }^{4}$ Department of Internal Medicine, ARNAS Civico Benfratelli Hospital, PROMISE, University of Palermo, Italy

\begin{abstract}
Atrial fibrillation (AF) and Heart Failure (HF) are evolving epidemies, together responsible for substantial human suffering and health-care expenditure. The simultaneous co-existence of the two conditions is associated with higher mortality rates than those observed in individuals with only one or none of them. Patients with concomitant HF and AF suffer from even worse symptoms and poorer prognosis, yet evidence-based evaluation and management of this group of patients is lacking.

In this review, we evaluate the common mechanisms for the development of AF in HF patients and vice versa, focusing on the evidence for potential treatment strategies. Recent data have suggested that these patients may respond differently if compared to those with HF or AF alone. These results highlight the clear clinical need to identify and treat these diseases according to best evidence, in order to prevent adverse outcomes and reduce the huge burden
\end{abstract}

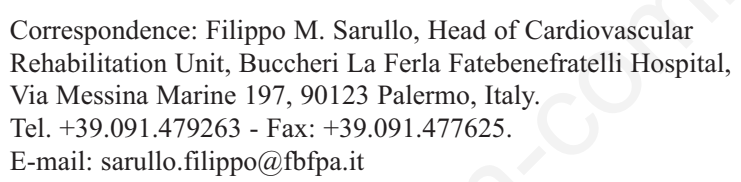

Key words: atrial fibrillation; heart failure; arrhythmia-induced cardiomyopathy.

Authors' contributions: All the authors made a substantive intellectual contribution. All the authors have read and approved the final version of the manuscript and agreed to be accountable for all aspects of the work.

Conflict of interests: The authors declare that they have no competing interests, and all authors confirm accuracy.

Conference presentation: SICGE 2019 congress.

Received for publication: 4 March 2020.

Accepted for publication: 18 April 2020.

${ }^{\circ}$ Copyright: the Author(s), 2020

Licensee PAGEPress, Italy

Monaldi Archives for Chest Disease 2020; 90:1264

doi: 10.4081/monaldi.2020.1264

This article is distributed under the terms of the Creative Commons Attribution Noncommercial License (by-nc 4.0) which permits any noncommercial use, distribution, and reproduction in any medium, provided the original author(s) and source are credited. that $\mathrm{HF}$ and $\mathrm{AF}$ are expected to have on global healthcare systems in the future.

\section{Introduction}

Atrial fibrillation (AF) is the most common arrhythmia in general population with higher prevalence in developed countries, in older patients and patients with different co-morbidities such as heart failure (HF) $[1,2]$. HF prevalence is approximately $1-2 \%$ of the adult population in developed countries, rising over $10 \%$ in people $>70$ years old [3].

Both AF and HF are growing problems, and the number of individuals who suffer from the two conditions concurrently is expanding as well. These conditions share common risk factors, and each has a propensity to cause the other [4]. Large HF trials have revealed that the likelihood of AF increases with the severity of HF [5], ranging from $10 \%$ in HF with NYHA class I to class II symptoms to $50 \%$ in HF with NYHA class IV symptoms.

EORP-AF registry has shown $47.5 \%$ of $\mathrm{HF}$ with reduced or preserved ejection fraction (HFrEF or HFpEF) in a large European cohort of AF patients [6]. Similarly, large AF trials record high $\mathrm{HF}$ prevalence rates, ranging from $21 \%$ to $68 \%$ [7].

Furthermore, AF is the most common arrhythmia in HFpEF, with a prevalence of $20 \%$ to $40 \%$ at the time of presentation [8]. It occurs in two-thirds of patients at some point during the course of HFpEF [9]. Patients with HFpEF are more likely to have prevalent $\mathrm{AF}$ or $\mathrm{AF}$ at any time compared with those with heart failure and reduced ejection fraction [9]. Right ventricular (RV) dysfunction and $\mathrm{AF}$ are common in patients with HFpEF; they often coexist and are independently associated with a poor prognosis [10].

Recent studies have indicated a potential relationship between $\mathrm{AF}$ and RV dysfunction in HFpEF [11,12]. For example, the prevalence of $\mathrm{AF}$ in patients without RV dysfunction ranges from $31 \%$ to $53 \%$, while it soars to $6 \%-73 \%$ in HFpEF patients with RV dysfunction [11-13]. Mitral regurgitation (MR) is one of the most common valvular disorders and it is the most common regurgitant disorder in patients with HFrEF and a known risk factor for AF [14].

However, the prevalence and pathophysiology of MR among patients with AF remains unexplored.

\section{Pathophysiology}

As highlighted by the epidemiological data, AF and HF tend to share the same population, risk factors and each one can cause 
the development of the other one. The reason of this relationship is explained by the pathophysiology of the two conditions: in fact, each disease condition induces structural, neuro-hormonal, and inflammatory changes that can predispose a patient to the other disease. The acute hemodynamic effects of AF are predominantly loss of atrial systole, which can further reduce cardiac index and acutely decompensate HF.

\section{AF and left atrial function}

Loss of reservoir, conduit and booster function of the left atrium (LA) is likely a consequence of the atrial fibrosis secondary to the increased wall stress, the inflammatory cytokines and the circulating neuro-hormonal factors seen in both HFrEF and HFpEF $[15,16]$.

In HF patients, loss of "atrial kick," changes in LA mechanics, loss of reservoir, conduit, and booster functions may impact on patient functional status as well as adversely affecting outcomes. Loss of atrial systole decreases cardiac output by up to $25 \%$ and this plays a significant role, particularly in diastolic dysfunction $[17,18]$.

\section{High ventricular rate and $\mathrm{AF}$-induced heart rate variability}

The high ventricular rate in AF reduces the filling time and the end diastolic volume determining a reduction in stroke volume; moreover, the irregularity of cardiac cycles alters the normal filling and emptying timing, contributing to ventricular remodeling and to impairing systolic function [15].

The reduction in stroke volume and the heart rate variability also induce an increase in neuro-humoral activation, with an incremented concentration of cardiovascular markers, for example high-sensitivity troponin $\mathrm{T}$ (hsTnT) or N-terminal pro-brain natriuretic peptide (NT-proBNP), that play a prognostic role especially in patients with $\mathrm{HF}[19,20]$.

\section{AF and renin-angiotensin-aldosterone system}

Upregulation of the renin-angiotensin-aldosterone system (RAAS) axis is thought to promote atrial fibrosis. In particular, Angiotensin II has been shown to stimulate cardiac fibroblast proliferation. This acts synergistically with oxidative stress and cytokines such as interleukin-6 and tumor necrosis factor (TNF) to induce fibrosis.

There can be seen the existence of an imbalance of the RAAS axis with LV dysfunction that promotes physiological maladaptation, increasing filling pressures and afterload. Stretching of the myocardium results in fibrosis and conduction abnormalities [21,22].

\section{AF and arrhythmia-induced cardiomyopathy}

Persistent AF can lead to arrhythmia-induced cardiomyopathy (AIC), which is a condition characterized by a dilated cardiomyopathy (increased LV end-diastolic dimension and area) with moderate to severe biventricular systolic dysfunction, normal LV septal and posterior wall thickness (lack of hypertrophy). Mitral insufficiency may be present due to LV and mitral annular dilatation with lack of leaflet coaptation.

The risk of developing AIC depends not only on the type, but also on the duration and rate of tachycardia. It should be suspected in patients with mean heart rate $>100$ beats $/ \mathrm{min}$, atrial fibrillation, and/or premature ventricular contractions burden $\geq 10 \%$ [23].

The process is mediated by changes in cellular and neuro-hormonal factors and extracellular remodeling as well. Resting tachycardia, increased HR with exercise, and irregularities in ventricular rhythm result in alterations of myocardial gene and protein expression, calcium handling, and increased sympathetic discharge with detrimental effects on ventricular function $[15,17,18,23]$. Recovery of ejection fraction (EF) after cardioversion or rate control confirms AIC.

In the same way enalapril has demonstrated to reduce atrial inflammation, fibrosis, remodeling and mean duration of $\mathrm{AF}$ in a similar population $[24,25]$. Furthermore, atrial scarring and reduced electrical activity can also derive from mechanical stretch of atrial wall, as observed in human patients comparing data from electro-anatomical mapping and CT scan [26].

\section{AF and mitral regurgitation}

MR is classified as primary (organic), when the mitral leaflets or the subvalvular apparatus are structurally abnormal causing leaflet malcoaptation, or secondary (functional) when the leaflets and subvalvular apparatus are normal and leaflet malcoaptation is caused by global or regional left ventricular (LV) remodeling that displace the papillary muscles, tethering the mitral leaflets, or by reduction of LV closing forces.

$\mathrm{AF}$ is a common sequela of degenerative mitral regurgitation (DMR) and it can lead to progressive left ventricular failure if untreated [27]. DMR may lead to the development of AF via left atrial (LA) volume and pressure overload, progressive atrial fibrosis, LA enlargement, and electroanatomic remodeling [2,27].

Progressive LA enlargement and remodeling - hallmarks of long-standing DMR - promote AF substrate by affecting cell coupling, altering conduction velocity, and promoting reentry [27].

\section{Prognosis}

The prognostic implications of AF development in HF is still a controversial subject. Older trials, such as the Vasodilator-Heart Failure Trial (V-HeFT), reported no difference in mortality between patients with mild-to-moderate HF in sinus rhythm (SR) or with the development of AF [28-30].

Retrospective analysis of the data from the Studies of Left Ventricular Dysfunction (SOLVD) trial looking at the association between $\mathrm{AF}$ and mortality showed that patients with LV dysfunction and AF at baseline had higher all-cause mortality and death from pump failure. The risk of arrhythmic death was comparable among patients with SR vs AF. Compared to SR, patients with AF were older, more likely to be NYHA functional class III-IV and with a lower mean left ventricular ejection fraction (LVEF) [31].

Similarly, results from the large randomized controlled trial of Candesartan in Heart Failure-Assessment of Reduction in Mortality and Morbidity (CHARM) indicated that baseline AF in patients with symptomatic HF conferred increased morbidity and mortality irrespective of ejection fraction (EF). Furthermore, the development of new-onset AF resulted in increased absolute risk for adverse cardiovascular outcomes in patients with HFrEF and greater relative risk of cardiovascular death and HF hospitalization in those with preserved LV function [32].

An analysis of patients enrolled in the Valsartan in Acute Myocardial Infarction (VALIANT) trial [33] revealed a high mortality rate in patients who developed AF with post-MI cardiac dysfunction. The authors reported occurrence of AF in the peri-infarct period with LV dysfunction ranges from 5\%-21\% and underlined how these patients tend to have higher mortality and stroke rates, both in-hospital and following discharge. However, limited data are available about a rate or rhythm control strategy in the periinfarct period as a possible influence factor in-hospital and longterm outcomes [33-35]. 
A contemporary diagnosis of HF impairs the prognosis of AF patients: in EURObservational Research Programme Pilot survey on atrial fibrillation (EORP-AF Pilot) the thrombo-embolic risk at 1 year was $4 \%$ in AF-only patients $v s$ 13.4\% in AF with HF patients with non-significant differences between HFrEF vs. HFpEF. In the same way, mortality at 1 year was $3 \%$ in AF-only patients vs $10.7 \%$ in AF with HF patients, without significant difference between HFrEF and HFpEF [6].

From another prospective, AF predicts a poorer prognosis in HF patients: in a contemporary population of HFrEF from PARADIGM-HF and ATMOSPHERE, 35.6\% had history of AF. The risk of primary endpoint (a composite of cardiovascular death, HF hospitalization, all-cause mortality and stroke) was higher in patients with paroxysmal AF than in patients without $\mathrm{AF}$, due to an increased risk of HF hospitalization and stroke.

Furthermore, patients who developed new-onset AF were older, mostly male and were at higher overall risk of cardiovascular mortality and HF hospitalization [35]. Interestingly, the risk of HF hospitalization was not so high in patients with persistent/permanent AF, probably according to the fact that AF paroxysms represent a marker of HF instability or that patients with persistent/permanent $\mathrm{AF}$ receive more treatments to control the ventricular rate [35].

Dysfunctions of both ventricles often coexist in CHF patients. LV failure can lead to right ventricular (RV) dysfunction. Ventricular interdependence implies the fact that dysfunctions of both ventricles frequently coexist. Many studies showed that in patients with advanced $\mathrm{CHF}$ and $\mathrm{LVEF}<40 \%$ prognosis strongly depends on RV function. RV function influenced the total outcome in these patients more significantly than LV function [36,37].

In patients with $\mathrm{AF}, \mathrm{LV}$ diastolic function is often worse and may result in deterioration of RV function [38]. In fact, RV function is determined by the heart rhythm, RV filling time, RV systolic synchrony and interdependence between both ventricles. Maintenance of sinus rhythm and atrio-ventricular synchrony is crucial for RV function especially in chronic and acute RV failure. In patients with $\mathrm{HFpEF}, \mathrm{RV}$ and RA function are more depressed in AF than in sinus rhythm patients. This association was independent from afterload. Moreover, patients in sinus rhythm who had earlier AF also displayed more RV and RA dysfunction than patients without any history of AF. Furthermore, reduced RA function was strongly and independently related to RV dysfunction in HFpEF [12].

\section{Treatment}

AF management includes two different aspects: prevention of stroke with anticoagulation therapy and management of cardiac rhythm or ventricular rate.

\section{Anticoagulation therapy}

Considering the high risk of stroke in AF population, anticoagulation represents a cornerstone of therapy especially in patients with HF: HF, in facts, increases the risk of stroke in AF, both HFrEF and HFpEF (but the former more than the latter) [39] and it is considered in the $\mathrm{CHA}_{2} \mathrm{DS}_{2}$-VASc score.

As already seen, Mogensen et al. [36] found a higher risk of stroke in patients with paroxysmal AF and less use of anticoagulation despite as high $\mathrm{CHA}_{2} \mathrm{DS}_{2}$-VASc score versus persistent/permanent AF patients. This observation highlights the importance of adequate anticoagulation in patients with HF and paroxysmal AF.

\section{Rhythm control versus rate control strategy in AF}

According to data on prognostic impact of AF in HF, a rhythm control strategy seems intuitively preferable. However, data from the AFFIRM trial [40] demonstrates no difference in survival while using a rhythm control strategy versus a rate control strategy in a non-selected AF population (with appropriate anticoagulation).

Interestingly, neither a better quality of life can be reached using a rhythm control strategy instead of the rate control one [41].

Furthermore, the AF-CHF trial confirms these findings in a selected population with left ventricle systolic dysfunction (EF $<35 \%$ ) [42]. Therefore, we can consider both strategies when we approach a patient with concomitant $\mathrm{AF}$ and HF.

Concerning rhythm control strategy, many antiarrhythmic drugs with a good efficacy and low side effects are contraindicated in the setting of HF, but amiodarone can be safely used in these patients to restore sinus rhythm [43]. It can also reduce ventricular rate by 10-12 bpm after 8-12 h [44]. Dofetilide could have the indication for this purpose as well, but it is not available in Europe.

Another strategy to restore acutely sinus rhythm is electrical cardioversion, which is the method of choice in severely hemodynamically compromised patients with paroxysm or new onset AF in order to restore sinus rhythm [1].

Concerning rate control strategy, we have more options for pharmacological therapy. Beta-blockers are standard of care in HFrEF regardless of heart rhythm, for their known effects on reducing mortality and $\mathrm{HF}$ hospitalization. In the AF-CHF trials the use of beta-blockers resulted associated with a significant reduction in over-all mortality and cardiovascular mortality [45]. However, in a meta-analysis of 13 studies, the effects of beta blockers in AF and HFrEF patients was neutral on mortality and cardiovascular mortality [46]. Although this equivocal findings, beta-blockers still remain the first line therapy for rate control as indicated in European guidelines [1]. Non-dihydropyridine calcium channel blockers should be avoided in HFrEF because of their negative inotropic effect [47] but they could be used in HFpEF. Like beta-blockers, digoxin has a role in HFrEF in patients in sinus rhythm [48] but its use in AF is not supported by strong evidence. Moreover, according to the lack of strong evidence and its narrow therapeutic window, digoxin is still used and indicated by European Guidelines but the dosage has to be conservative and followed by plasma levels, especially in elderly and in patients with renal dysfunction [49].

Recent data from an open-label randomized trial of AF ablation in $\mathrm{HFrEF}$ patients with $\mathrm{EF}<35 \%$ showed that patients who were assigned to ablation had a reduced incidence of death or HF admissions with a rising trend in EF level post-ablation [50].

The benefit was seen with a decrease in the burden of AF from $60 \%$ of time with medical therapy to $25 \%$ with ablation, suggesting that a reduction in the amount of time in AF may be sufficient for clinical benefit.

\section{Mitral valve intervention}

Mitral valve intervention is indicated for symptomatic severe valvular disease (typically breathlessness and fatigue). It is also indicated for asymptomatic severe valvular disease with evidence of detrimental pathophysiological changes, such as left ventricular systolic dysfunction, pulmonary hypertension, or atrial fibrillation in asymptomatic severe mitral regurgitation. Percutaneous mitral commissurotomy (PMC) is indicated for severe mitral stenosis with favorable anatomical characteristics.

Mitral valve repair is preferred over valve replacement when feasible. Cases for the intervention should be discussed by a Heart 
Valve Team in order to recommend the best approach, e.g., PMC, full sternotomy or minimal access valve surgery, or newer less invasive techniques as these become established [51].

\section{Management of concomitant heart failure and reduced ejection fraction and atrial fibrillation}

Activation of neurohormonal pathways and RAAS are well described in HF, and the majority of evidence-based therapies target these compensatory mechanisms [52,53]. Angiotensin converting enzyme inhibitors (ACEi) have proven efficacy in HFrEF for significant reduction in mortality, sudden cardiac death, and HF hospitalization, but no trials have examined their benefit in concomitant AF.

Angiotensin receptor blockers (ARBs) are recommended as alternatives to ACEi in cases of intolerance, and there are numerous trials supporting their use in HFrEF [52].

In CHARM, randomization to candesartan significantly reduced $\mathrm{CV}$ death or HF hospitalization in HFrEF patients with concomitant $\mathrm{AF}$, similar to that observed in patients without $\mathrm{AF}$ at baseline [32].

In contrast, Irbesartan did not reduce the composite outcome of hospitalization due to HF, stroke, myocardial infarction, or death from vascular causes in AF patients enrolled in the Atrial Fibrillation Clopidogrel Trial with Irbesartan for Prevention of Vascular Events A or W trials [53].

\section{Physical activity and exercise training}

Physical activity and exercise training improve symptoms and they can have antiarrhythmic effects in individuals with paroxysmal AF and may be protective against the development of AF [54].

In patients with chronic heart failure with HFrEF, as shown in the "Heart Failure: A Controlled Trial Investigating Outcomes of Exercise Training (HF-ACTION) study", exercise training is associated with improved exercise capacity, improved quality of life, and reduced all-cause mortality and hospitalization [54].

\section{Conclusions}

$\mathrm{AF}$ and HF are increasing in general population and we will find them in the same patient more frequently, especially in elderly. Both conditions together affect the prognosis of patients and complicate the pharmacological management [55].

Despite the possibility of evidence-based therapy for HFrEF, beta-blockers and digoxin probably lose their prognostic effects in AF. However, an adequate therapy is required: it is almost mandatory to have anticoagulation (regarding score risks) also in paroxysmal AF and to reach rate or rhythm control especially in AIC patients who can recover from the ventricular dysfunction.

Exercise training reduced all-cause mortality, hospitalizations, and improve health status in HFrEF patients and permanent AF [54].

Further research is still required to improve treatment of HFpEF and to understand better its relationship with AF.

\section{References}

1. Kirchhof P, Benussi S, Kotecha D, et al. 2016 ESC Guidelines for the management of atrial fibrillation developed in collaboration with EACTS. Eur Heart J 2016;37:2893-62.

2. Zoni-Berisso M, Lercari F, Carazza T, Domenicucci S.
Epidemiology of atrial fbrillation: European perspective.C lin Epidemiol 2014;6:213-20.

3. Ponikowski P, Voors AA, Anker SD, et al. 2016 ESC Guidelines for the diagnosis and treatment of acute and chronic heart failure. Eur Heart J 2016;37:2129-200.

4. Psaty BM, Manolio TA, Kuller LH, et al. Incidence of and risk factors for atrial fibrillation in older adults. Circulation 1997;96:2455-61.

5. Maisel WH, Stevenson LW. Atrial fibrillation in heart failure: epidemiology, pathophysiology and rationale for therapy. Am J Cardiol 2003;91:2D-8.

6. Lip GYH,Laroche C, Popescu MI, et al. Heart failure in patients with atrial fibrillation in Europe: A report from the EURObservational Research Programme Pilot survey on Atrial Fibrillation. Eur J Heart Fail 2015;17:570-82.

7. Gomez-Outes A, Terleira-Fernadez AI, Calvo-Rojas G, et al. Dabigatran, rivaroxaban, or apixaban versus warfarin in patients with nonvalvular atrial fibrillation: a systematic review and meta-analysis of subgroups. Thrombosis 2013;640:723.

8. Lam CS, Donal E, Kraigher-Krainer E, Vasan RS. Epidemiology and clinical course of heart failure with preserved ejection fraction. Eur J Heart Fail 2011;13:18-28.

9. Linderer T, Chatterjee K, Parmley WW, et al. Influence of atrial systole on the Frank-Starling relation and the end-diastolic pressure-diameter relation to the left ventricle. Circulation 1983;67:1045-53.

10. Santhanakrishnan R, Wang N, Larson MG, et al.Atrial fibrillation begets heart failure and vice versa: temporal associations and differences in preserved reduced ejection fraction. Circulation 2016;133:484-92.

11. Gorter TM, Hoendermis ES, van Veldhuisen DJ, et al. Right ventricular dysfunction in heart failure with preserved ejection fraction: a systematic review and meta-analysis. Eur J Heart Fail 2016;18:1472-87.

12. Melenovsky V, Hwang SJ, Lin G, et al. Right heart dysfunction in heart failure with preserved ejection fraction. Eur Heart J 2014;35:3452-62.

13. Gorter TM, Van Melle JP, Rienstra M, et al. Right heart dysfunction in heart failurewith preserved ejection fraction: the impact of atrial fibrillation. J Cardiac Fail 2018;24:177-85.

14. Lam CS, Rienstra M, Tay WT, et al. Atrial fibrillation in heart failure with preserved ejection fraction: association with exercise capacity, left ventricular filling pressures, natriuretic peptides, and left atrial volume. JACC Heart Fail 2017;5:92-8.

15. Nkomo VT, Gardin JM, Skelton TN, et al. Burden of valvular heart diseases: a population-based study. Lancet 2006;368: 1005-11.

16. Ling LH, Kistler PM, Kalman JM, et al. Comorbidity of atrial fibrillation and heart failure. Nat Rev Cardiol 2016;13:131-47.

17. Kotecha D, Piccini JP. Atrial fibrillation in heart failure: what should we do? Eur Heart J 2015;36:3250-7.

18. Redfield MM, Kay GN, Jenkins LS, et al. Tachycardia-related cardiomyopathy: a common cause of ventricular dysfunction in patients with atrial fibrillation referred for atrioventricular ablation. Mayo Clin Proc 2000;75:790-5.

19. Patel RB, Vaduganathan M, Shah SJ, Butler J. Atrial fibrillation in heart failure with preserved ejection fraction: insights into mechanisms and therapeutics. Pharmacol Ther 2017;176:32-9.

20. Latini R, Masson S, Pirelli S, et al. Circulating cardiovascular biomarkers in recurrent atrial fibrillation: data from the GISSIAtrial Fibrillation Trial. J Internal Med 2011;269:160-71.

21. Pandey A, Kim S, Moore C, et al. Predictors and prognostic 
implications of incident heart failure in patients with prevalent atrial fibrillation. JACC Heart Fail 2017;5:44-52.

22. Melenovsky V, Hwang SJ, Lin G, et al. Right heart dysfunction in heart failure with preserved ejection fraction. Eur Heart $\mathrm{J}$ 2014;35:3452-62.

23. Gopinathannair R, Etheridge SP, Marchlinski FE, et al. Arrhythmia-induced cardiomyopathies: mechanisms, recognition, and management. J Am Coll Cardiol 2015;66:1714-28.

24. Huizar JF, Ellenbogen KA, Tan AY, Kaszala K. Arrhythmiainduced cardiomyopathy: JACC state-of-the-art review. J Am Coll Cardiol 2019;73:2328-44.

25. Li D, Shinagawa K, Pang L, et al. Effects of angiotensin-converting enzyme inhibition on the development of the atrial fibrillation substrate in dogs with ventricular tachypacing - Induced congestive heart failure. Circulation 2001;104:2608-14.

26. Hunter RJ, Liu Y, Lu Y, et al. Left atrial wall stress distribution and its relationship to electrophysiologic remodeling in persistent atrial fibrillation. Circ Arrhythmia Electrophysiol 2012;5:351-60.

27. Borger MA, Mansour MC, Levine RA.Atrial fibrillation and mitral valve prolapse time to intervene? J Am Coll Cardiol 2019;73:275-7.

28. Smith JG, Newton-Cheh C, Almgren P, et al. Assessment of conventional cardiovascular risk factors and multiple biomarkers for the prediction of incident heart failure and atrial fibrillation. J Am Coll Cardiol 2010;56:1712-9.

29. Carson PE, Johnson GR, Dunkman WB, et al. The influence of atrial fibrillation on prognosis in mild to moderate heart failure. The V-HeFT Studies. The V-HeFT VA Cooperative Studies Group. Circulation 1993;87:102-10.

30. Anter E, Jessup M, Callans DJ. Atrial fibrillation and heart failure: treatment considerations for a dual epidemic. Circulation 2009; 119:2516-25.

31. Dries DL, Exner DV, Gersh BJ, et al. Atrial fibrillation is associated with an increased risk for mortality and heart failure progression in patients with asymptomatic and symptomatic left ventricular systolic dysfunction: a retrospective analysis of the SOLVD trials. Studies of Left Ventricular Dysfunction. J Am Coll Cardiol 1998;32:695-703.

32. Olsson LG, Swedberg K, Ducharme A, et al. Atrial fibrillation and risk of clinical events in chronic heart failure with and without left ventricular systolic dysfunction: results from the Candesartan in Heart failure-Assessment of Reduction in Mortality and morbidity (CHARM) program. J Am Coll Cardiol 2006;47:1997-2004.

33. Nilsson KR, Jr, Al-Khatib SM, Zhou Y, et al. Atrial fibrillation management strategies and early mortality after myocardial infarction: results from the Valsartan in Acute Myocardial Infarction (VALIANT) Trial. Heart 2010;96:838-42.

34. Lehto M, Snapinn S, Dickstein K, et al. Prognostic risk of atrial fibrillation in acute myocardial infarction complicated by left ventricular dysfunction: the OPTIMAAL experience. Eur Heart J 2005;26:350-6.

35. Pedersen OD, Bagger H, Køber L, Torp-Pedersen C. The occurrence and prognostic significance of atrial fibrillation/flutter following acute myocardial infarction. TRACE Study Group. TRAndolapril Cardiac Evalution. Eur Heart J 1999;20:748-54.

36. Mogensen UM, Jhund PS, Abraham WT, et al. "Type of atrial fibrillation and outcomes in patients with heart failure and reduced ejection fraction. J Am Coll Cardiol 2017;70: 2490-500.

37. Meluzin J, Spinarova L, Hude, et al. Prognostic importance of various echocardiographic right ventricular functional parame- ters in patients with symptomatic heart failure. J Am Soc Echocardiogr 2005;18:435-44.

38. Majos E, Dąbrowski R, Szwed H. The right ventricle in patients with chronic heart failure and atrial fibrillation. Cardiol J 2013;20:220-6.

39. Fung JW, Sanderson JE, Yip GW, et al. Impact of atrial fibrillation in heart failure with normal ejection fraction: A clinical and echocardiographic study. J Card Fail 2007;13:649-6.

40. Jenkins LS, Brodsky M, Schron E, et al. Quality of life in atrial fibrillation: the Atrial Fibrillation Follow-up Investigation of Rhythm Management (AFFIRM) study. Am Heart J 2005;149: $112-20$.

41. Wyse DG, Waldo AL, DiMarco JP, et al. A comparison of rate control and rhythm control in patients with atrial fibrillation. $\mathrm{N}$ Engl J Med 2002;347:1825-33.

42. McMurray JJV, Ezekowitz JA, Lewis BS, et al. Left ventricular systolic dysfunction, heart failure, and the risk of stroke and systemic embolism in patients with atrial fibrillation insights from the ARISTOTLE trial. Circ Heart Fail 2013;6:451-60.

43. Roy D, Talajic M, Nattel S, et al. Rhythm control versus rate control for atrial fibrillation and heart failure. N Engl J Med 2008;358:2667-77.

44. Connolly SJ, Cairns J, Gent M, et al. Effect of prophylactic amiodarone on mortality after acute myocardial infarction and in congestive heart failure: Meta-analysis of individual data from 6500 patients in randomised trials. Lancet 1997;350:1417-24.

45. Chevalier P, Durand-Dubief, Burri AH, et al. Amiodarone versus placebo and class IC drugs for cardioversion of recentonset atrial fibrillation: A meta-analysis. J Am Coll Cardiol 2003;41:255-62.

46. Talajic M,Khairy P, Levesque S, et al. Maintenance of sinus rhythm and survival in patients with heart failure and atrial fibrillation. J Am Coll Cardiol 2010;55:1796-802.

47. Goldstein RE,Boccuzzi SJ, Cruess D, Nattel S. Diltiazem increases late-onset congestive heart failure in postinfarction patients with early reduction in ejection fraction. Circulation 1991;83:52-60.

48. Garg R, Gorlin R, Smith T, Yusuf S. The effect of digoxin on mortality and morbidity in patients with heart failure. N Engl J Med 1997;336:525-33.

49. Reynolds MR. Outcomes with digoxin in atrial fibrillation: more data, no answers. J Am Coll Cardiol 2014;64:669-71.

50. Marrouche NF, Brachmann J, Andresen D, et al. CASTLE-AF Investigators. Catheter ablation for atrial fibrillation with heart failure. N Engl J Med 2018;378:417-27.

51. Falk V, Baumgartner H, Bax JJ, et al. ESC Scientific Document Group. 2017 ESC/EACTS Guidelines for the management of valvular heart disease. Eur J Cardiothorac Surg 2017;52:616-64.

52. Ponikowski P, Voors AA, Anker SD, et al. 2016 ESC Guidelines for the diagnosis and treatment of acute and chronic heart failure: The Task Force for the Diagnosis and Treatment of Acute and Chronic Heart Failure of the European Society of Cardiology (ESC). Developed in collaboration with the Heart Failure Association (HFA) of the ESC. Eur Heart J 2016;37:2129-200.

53. ACTIVE I Investigators, Yusuf S, Healey JS, et al. Irbesartan in patients with atrial fibrillation. N Engl J Med 2011;364:928-38.

54. Malmo V, Nes BM. Amundsen BH, et al. Aerobic interval training reduces the burden of atrial fibrillation in the short term: a randomized trial. Circulation 2016;133:466-73.

55. Fumagalli S, Pelagalli G, Migliorini M, et al. The complex interactin between atrial fibrillation and heart failure in enderly patients. Monaldi Arch Chest Dis 2019;89:1050. 